\title{
Sentinel Lymph Node Biopsy: From B04 to Tumor Genomics
}

\author{
Jean Bao, MD and Armando E. Giuliano, MD \\ Cedars-Sinai Medical Center, Los Angeles, CA
}

In 1974, Giuliano et al. reported the feasibility of intraoperative sentinel lymph node biopsy (SLNB) with lymphatic mapping using isosulfan blue dye in 170 patients. ${ }^{1}$ Since then, many prospective trials have validated this technique as safe and accurate, establishing SLNB as the standard axillary staging procedure in clinically node-negative women even before any randomized trials or long-term outcomes were reported. Historically, the false-negative rate (FNR) of SLNB ranged between 5 and $10 \%$ even in experienced hands, but has been reported as high as $16.7 \% .^{2-5}$ FNRs have decreased as the operation became more common and widely taught. Notably, axillary recurrence rates are much lower than reported FNRs; the incidence of axillary recurrence following a negative SLNB varies from 0.2 to $1 \%{ }^{5-8}$ In this issue of Annals of Surgical Oncology, Matsen et al. contribute to our understanding of axillary recurrence by demonstrating in their large study an axillary recurrence rate of only $0.9 \%$ at 10 and 15 years after a negative SLNB. This is one of the longer follow-up times of any published study on SLNB, leading the authors to conclude that SLNB remains a safe and effective axillary staging technique.

The National Surgical Adjuvant Breast and Bowel Project (NSABP)-B32 trial and the study by Veronesi et al. are two large, prospective, randomized trials evaluating SLNB in clinically node-negative patients with more than 7 years of follow-up. NSABP-B32 reported a SLNB FNR of $9.8 \%$, with a regional recurrence rate of only $0.7 \%$ among those who had SLNB alone at 95.6 months. ${ }^{6}$ Veronesi et al. demonstrated similar results, with only two axillary recurrences in the SLNB-only arm at 102 months of follow-up, a relapse rate of $0.8 \%{ }^{8}$ Incidentally, both of

(C) Society of Surgical Oncology 2016

First Received: 22 March 2016;

Published Online: 13 April 2016

A. E. Giuliano, MD

e-mail: Armando.Giuliano@cshs.org these axillary recurrences occurred more than 5 years after the initial procedure. The Sentinella trial reported one of the highest FNRs of SLNB, at $16.7 \% .^{5}$ Despite this, there was only one axillary recurrence at 56 months. With such low regional recurrence rates and equivalent survival to axillary lymph node dissection (ALND), there is no question that axillary dissection is unnecessary when the sentinel lymph node (SLN) is free of tumor.

The utility of axillary dissection and extensive chestwall irradiation were examined 40 years ago when the NSABP-B04 study compared radical mastectomy with total mastectomy with and without chest wall radiotherapy. ${ }^{9}$ No statistically significant survival difference was found among the three groups of clinically node-negative women. Nearly $40 \%$ of the radical mastectomy patients in NSABP-B04 had pathologically confirmed nodal disease. In a randomized trial, it may be assumed that approximately $40 \%$ of the mastectomy-only patients were also node-positive. Overall survival at 10 years was only $57 \%$, a dismal number compared with the $90 \%$ survival rate in the modern era. However, what was remarkable about the B04 study was that less than half the number of expected node-positive patients in the mastectomy group developed axillary failure, even as late as 25 years of follow-up. This is much less than what would have been expected, especially considering that these women had large palpable breast tumors, had no targeted axillary treatment, and received no adjuvant systemic therapy, not even tamoxifen.

The results of the more recent American College of Surgeons Oncology Group (ACOSOG) Z0011 trial not only question the utility of removing additional axillary lymph nodes beyond the SLN with metastatic disease but also, more importantly, underscore the importance of modernday multimodality therapy. ${ }^{10}$ At 6.3 years of follow-up, axillary relapse occurred in only $0.9 \%$ of patients who had SLN metastasis but no axillary dissection. Twenty-seven percent of them should have had additional lymph node metastases since that was seen in the ALND arm of the 
study. It is important to keep in mind that the Z0011 patients all had whole-breast irradiation (WBI) following breastconserving surgery, and the vast majority had adjuvant systemic therapy, as did the patients in B32 and the study by Veronesi et al. The cohort in the study by Matsen et al., $90 \%$ of whom had T1 tumors, were nearly all treated with breast conservation and WBI $(85 \%)$.

All these data suggest not all residual axillary disease will progress to become clinically evident with regional relapse or death from breast cancer. Chemotherapy and hormonal therapy are known to reduce locoregional recurrence, and it is likely residual nodal disease is eradicated or sterilized by systemic therapy. In addition, tangential breast irradiation fields oftentimes cover the SLNB field and perhaps much of level 1, or even level 2, of the axilla, hence treating most axillary disease that may be left behind. The B04 mastectomy-only patients did not undergo any of the above adjuvant strategies; however, their axillary recurrence was not as high as would have been predicted by the number of radical mastectomy patients with positive nodes. Perhaps some residual nodal disease simply remains clinically dormant for reasons not well understood. One possible explanation is that only a small proportion of lymph node metastases contain cancer stem cells, whereas the majority do not, and perhaps only the cancer stem cells have the ability to propagate, disseminate, and survive elsewhere in the body. The biology of nodal metastasis may only permit these cancer cells to grow in lymph nodes but not disseminate. Gene expression analysis is increasingly utilized to further characterize tumor biology beyond what may be predicted by anatomic classifications and clinicopathologic factors. Matsen et al. point out that concerns for late axillary recurrence, especially among women with estrogen receptor-positive breast cancer, may be unfounded. Although this may be true, the risk for distant recurrence remains realistic at 1.5-1.8\% per year for up to 10 years after 5 years of hormonal therapy, and should not be overlooked. ${ }^{11}$

Enhanced pathologic evaluation of SLNs has led to increased rates of detection of small-volume nodal disease, namely micrometastases and isolated tumors cells. The prognostic significance of micrometastases has been largely debated as older retrospective data associated micrometastases with worse survival. ${ }^{12-14}$ The cohort in the study by Matsen et al. most likely included some women with SLN micrometastases in the SLN-positive arm as their study was initiated prior to the 7th edition of the American Joint Committee on Cancer (AJCC) TNM staging system, which incorporated micrometastases into the staging classification. In the past few years, several large, prospective studies demonstrated that SLN micrometastasis is associated with low regional recurrence (1\% or less) and equivalent survival, and does not warrant axillary dissection. ${ }^{15,16}$ Furthermore, the analysis by Mittendorf et al. of over 8000 patients from MD Anderson and the ACOSOG Z0010 cohort showed no statistically significant difference in recurrence-free survival, disease-free survival, or overall survival between stage $1 \mathrm{~A}$ and stage $1 \mathrm{~B}$ patients. ${ }^{17}$ Despite the increased use of adjuvant systemic therapy in the stage 1B group, the study by Mittendorf et al. questions the current staging nomenclature, which is limited by anatomic classification without accounting for tumor biology.

As treatment becomes more individualized based on tumor genomics, the extent of nodal surgery may not alter therapy or prognosis. We also begin to question the utility of SLNB in certain cases. The European SOUND trial (Sentinel node vs. Observation after axillary UltrasouND) is underway to examine recurrence and survival rates among patients with clinical T1N0 breast cancer with negative preoperative axillary ultrasound but no surgical axillary staging, compared with those who receive SLNB. ${ }^{18}$

Management of breast cancer has made tremendous strides from 40 years ago when radical mastectomy failed to show a survival advantage, to today when axillary dissection can be omitted in some patients with positive SLNs without compromising locoregional control or survival. The importance of multimodality therapy certainly must not be understated. Therapeutic plans should be formulated based on not only the volume of nodal disease but also the genetic signature of the tumor. Thus, the long-term results of Matsen et al. reinforce the role of SLNB as a safe and effective axillary staging procedure among clinically nodenegative patients.

Disclosures None.

\section{REFERENCES}

1. Giuliano AE, Kirgan DM, Guenther JM, Morton DL. Lymphatic mapping and sentinel lymphadenectomy for breast cancer. Ann Surg. 1994;220:391-98.

2. Canavese G, Catturich A, Vecchio C, et al. Sentinel node biopsy compared with complete axillary dissection for staging early breast cancer with clinically negative lymph nodes: results of randomized trial. Ann Oncol. 2009;20:1001-07.

3. Krag DN, Anderson SJ, Julian TB, et al. Technical outcomes of sentinel-lymph-node resection and conventional axillary-lymphnode dissection in patients with clinically node-negative breast cancer: results from the NSABP B-32 randomised phase III trial. Lancet Oncol. 2007;8:881-8.

4. Veronesi U, Paganelli G, Viale G, et al. Sentinel lymph node biopsy and axillary dissection in breast cancer: results in a large series. J Natl Cancer Inst. 1999;91:368-73.

5. Zavagno G, De Salvo GL, Scalco G, et al. A randomized clinical trial on sentinel lymph node biopsy versus axillary lymph node dissection in breast cancer: results of the Sentinella/GIVOM trial. Ann Surg. 2008;247:207-13. 
6. Krag DN, Anderson SJ, Julian TB, et al. Sentinel-lymph-node resection compared with conventional axillary-lymph-node dissection in clinically node-negative patients with breast cancer: overall survival findings from the NSABP B-32 randomised phase 3 trial. Lancet Oncol. 2010;11:927-33.

7. Mansel RE, Fallowfield L, Kissin M, et al. Randomized multicenter trial of sentinel node biopsy versus standard axillary treatment in operable breast cancer: the ALMANAC Trial. $J$ Natl Cancer Inst. 2006;98:599-09.

8. Veronesi U, Viale G, Paganelli G, et al. Sentinel lymph node biopsy in breast cancer: ten-year results of a randomized controlled study. Ann Surg. 2010;251:595-600.

9. Fisher B, Jeong JH, Anderson S, et al. Twenty-five-year followup of a randomized trial comparing radical mastectomy, total mastectomy, and total mastectomy followed by irradiation. $N$ Engl J Med. 2002;347:567-75.

10. Giuliano AE, Hunt KK, Ballman KV, et al. Axillary dissection vs no axillary dissection in women with invasive breast cancer and sentinel node metastasis: a randomized clinical trial. JAMA. 2011;305:569-75.

11. Cuzick J, Sestak I, Baum M, et al. Effect of anastrozole and tamoxifen as adjuvant treatment for early-stage breast cancer: 10year analysis of the ATAC trial. Lancet Oncol. 2010;11:1135-41.

12. Chen SL, Hoehne FM, Giuliano AE. The prognostic significance of micrometastases in breast cancer: a SEER population-based analysis. Ann Surg Oncol. 2007;14:3378-84.
13. Cote RJ, Peterson HF, Chaiwun B, et al. Role of immunohistochemical detection of lymph-node metastases in management of breast cancer. International Breast Cancer Study Group. Lancet. 1999;354:896-900.

14. de Boer M, van Deurzen CH, van Dijck JA, et al. Micrometastases or isolated tumor cells and the outcome of breast cancer. $N$ Engl J Med. 2009;361:653-63.

15. Galimberti V, Cole BF, Zurrida S, et al. Axillary dissection versus no axillary dissection in patients with sentinel-node micrometastases (IBCSG 23-01): a phase 3 randomised controlled trial. Lancet Oncol. 2013;14:297-305.

16. Giuliano AE, Hawes D, Ballman KV, et al. Association of occult metastases in sentinel lymph nodes and bone marrow with survival among women with early-stage invasive breast cancer. JAMA. 2011;306:385-93.

17. Mittendorf EA, Hunt KK, Boughey JC, et al. Incorporation of sentinel lymph node metastasis size into a nomogram predicting nonsentinel lymph node involvement in breast cancer patients with a positive sentinel lymph node. Ann Surg. 2012;255:109-15.

18. Gentilini O, Veronesi U. Abandoning sentinel lymph node biopsy in early breast cancer? A new trial in progress at the European Institute of Oncology of Milan (SOUND: Sentinel node vs Observation after axillary UltraSouND). Breast. 2012;21:678-81. 